\title{
Anti-apoptotic and myocardial protective effects of ethyl pyruvate after regional ischaemia/reperfusion myocardial damage in an in vivo rat model
}

\author{
Haeng Seon Shim ${ }^{1}, \mathrm{MD}, \mathrm{PhD}$, Wang Gyu $\underline{\mathrm{Lee}}^{1}, \mathrm{MD}$, Yeon A $\underline{\mathrm{Kim}}^{2}$, MD, Jeong Yeol $\underline{\mathrm{Han}}^{2}$, MD, Miyeong Park ${ }^{2}$, MD, PhD,
} Yun Gyu Song ${ }^{3}$, MD, PhD, Joon Soo $\underline{\mathrm{Kim}}^{4, *}$, MD, PhD, II-Woo $\underline{\operatorname{Shin}}^{5}$, , MD, PhD

\begin{abstract}
INTRODUCTION The integration of reactive oxygen species is strongly associated with important pathophysiological mechanisms that mediate myocardial ischaemia/reperfusion (I/R) damage. Pyruvate is an efficacious scavenger of reactive oxygen species and a previous study has shown that ethyl pyruvate (EP) has a myocardial protective effect against regional I/R damage in an in vivo rat model. The purpose of this study was to determine whether the myocardial protective effect of EP is associated with anti-apoptosis.

METHODS Rats were allocated to receive EP dissolved in lactated Ringer's solution or lactated Ringer's solution alone, via intraperitoneal infusion one hour before ischaemia. They were exposed to 30 minutes of ischaemia followed by reperfusion of the left coronary artery territory over two hours. Anti-apoptotic effects were checked using several biochemical parameters after two hours of reperfusion. Apoptosis was analysed using measured caspase-3 activity, Western blotting of B-cell lymphoma 2 (Bcl-2) family protein cleaved by caspase-3, and assessment of DNA laddering patterns and the terminal deoxynucleotidyl transferase dUTP nick end labelling (TUNEL) staining test.

RESULTS In ischaemic myocardium, EP increased Bcl-2 expression, but reduced Bcl-2-associated X protein and cleaved caspase-3 expressions. EP reduced the expression of DNA laddering and the number of myocardial I/R-damaged TUNELpositive cells.

CONCLUSION This study demonstrated that EP has an anti-apoptotic effect after regional I/R damage in an in vivo rat heart model. The myocardial protective effect of EP may be related to its anti-apoptotic effect.
\end{abstract}

Keywords: apoptosis, ethyl pyruvate, myocardium, reperfusion injury

\section{INTRODUCTION}

Ischaemia/reperfusion (I/R) damage to the heart is a significant risk faced in many clinical procedures, including coronary thrombolytic therapy, percutaneous transluminal coronary angioplasty, on-pump cardiac surgery, coronary artery bypass graft and heart transplantation. During these interventions or surgeries, it is important to select appropriate components for the fluid used in order to reduce $\mathrm{I} / \mathrm{R}$ damage.

Pyruvate $\left(\mathrm{CH}_{3} \mathrm{COCOO}^{-}\right)$is the terminal outcome of glycolysis and the starting substrate for the tricarboxylic acid cycle. Pyruvate, a key intermediate in the oxidative and anaerobic metabolism of glucose, is a powerful scavenger of reactive oxygen species (ROS). ${ }^{(1-3)}$ The generation of ROS increases during both ischaemia and reperfusion, and ROS play a major role in the pathophysiology of intraoperative myocardial damage. ${ }^{(4)}$ It has been reported that pyruvate has hydroxyl radical $\left(\mathrm{OH}^{*}\right)$ scavenging(5) and organ protective effects in response to oxidative stress. ${ }^{(2,3,6)}$ The protective effects of pyruvate against oxidative stress have been reported in different organs, ${ }^{(7-13)}$ including the heart. ${ }^{(5,14,15)}$ However, pyruvate is unstable in aqueous solutions, and is inadequately soluble in water and saline. Ethyl pyruvate (EP) is very soluble in calcium solutions, forming a dimolecular complex with the enolate anion stabilised by divalent calcium cations. EP has far greater stability than pyruvate and may act as a pyruvate precursor.

EP is an efficacious anti-inflammatory and anti-apoptotic agent in in vitro and in vivo models. ${ }^{(16-25)} \mathrm{EP}$ is also effective in myocardial protection in a global I/R damage model. However, one type of myocardial damage commonly encountered in clinical conditions is regional I/R damage. In a previous study, we demonstrated the myocardial protective and anti-inflammatory effects of EP against I/R damage in an in vivo rat model. ${ }^{(19)}$ However, the anti-apoptotic effect of EP against I/R damage in an in vivo rat model has not been shown. Therefore, this study aimed to determine whether EP has an anti-apoptotic effect after regional $\mathrm{I} / \mathrm{R}$ damage in an in vivo rat model. A regional myocardial I/R damage model was used to compare the effects of lactated Ringer's solution (LR) and EP dissolved in LR on the various physiological and biochemical variables of apoptosis.

\section{METHODS}

Male Sprague-Dawley rats (Sam Tako Inc, Osan, South Korea) weighing between $200 \mathrm{~g}$ and $250 \mathrm{~g}$ each were used. All

\footnotetext{
${ }^{1}$ Department of Anesthesiology and Pain Medicine, Samsung Changwon Hospital, Sungkyunkwan University School of Medicine, ${ }^{2}$ Department of Anesthesiology and Pain Medicine, Gyeongsang National University Hospital, ${ }^{3}$ Department of Radiology, ${ }^{4}$ Department of Neurosurgery, Samsung Changwon Hospital, Sungkyunkwan University School of Medicine, Changwon, ${ }^{5}$ Department of Anesthesiology and Pain Medicine, Gyeongsang National University School of Medicine, Jinju, Gyeongsangnam-do, Republic of Korea *These two authors contributed equally to this work.

Correspondence: Dr II-Woo Shin, Professor, Department of Anesthesiology and Pain Medicine, Gyeongsang National University Hospital, Institute of Health Sciences, Gyeongsang National University School of Medicine, 79 Gangnam-ro, Jinju, 660-702, Republic of Korea. ilwooshin@gnu.ac.kr
} 
animals were preserved in accordance with the Guidance for the Description of Animal Research in Scientific Publications ${ }^{(26)}$ issued by the Institute for Laboratory Animal Research, National Research Council, USA, in 2011. The animals were prepared and surgery was performed as described in a previous report. ${ }^{(27)}$

General anaesthesia was performed with an intramuscular injection of $15 \mathrm{mg} / \mathrm{kg}$ tiletamine/zolazepam (Zoletil $®$ 50; Virbac Lab, Carros, France) and $8 \mathrm{mg} / \mathrm{kg}$ xylazine (Rompun ${ }^{\circledR}$; Bayer Korea, Seoul, South Korea). Tail movement was used as an indication that the rat had awakened from anaesthesia. We also administered $7.5 \mathrm{mg} / \mathrm{kg}$ tiletamine/zolazepam and $4 \mathrm{mg} / \mathrm{kg}$ xylazine via intramuscular injections to maintain the level of anaesthesia. The rats were intubated and mechanically ventilated (tidal volume $8 \mathrm{~mL} / \mathrm{kg}$; respiratory rate 80 strokes/ minute) (Type 7025; Ugo Basile, Comerio, Italy). Arterial blood $\mathrm{pH}$ and gases were preserved within normal physiological limits ( $\mathrm{pH}$ range 7.35-7.45; partial pressure of carbon dioxide in arterial blood 35-40 mmHg; partial pressure of oxygen in arterial blood 70-100 mmHg). Body temperature was checked with a rectal thermometer (Sirecust 1260; Siemens Medical Electronics, Danvers, MA, USA) and maintained at $36^{\circ} \mathrm{C}-38^{\circ} \mathrm{C}$ using an electrical heating pad and lamp. Electrocardiography (ECG) leads were placed to monitor changes in myocardial ischaemia.

Left thoracotomy was carried out and the heart was exposed. For I/R research, a 4-0 black silk, non-absorbable suture (Mersilk; Ethicon Inc, Somerville, NJ, USA) was used to induce regional myocardial ischaemia in the left coronary artery territory. Coronary artery occlusion was performed by tightening and clamping. Reperfusion was performed by releasing the clamp. Myocardial ischaemia was monitored based on ischaemic ECG changes, regional epicardial cyanosis and arrhythmia. After 30 minutes of ischaemia, the reperfused myocardium was maintained for two hours. The disappearance of epicardial cyanosis and epicardial hyperaemia verified a successful reperfusion.

Each drug was injected intraperitoneally one hour before the ischaemic offence. We infused the drugs into the intraperitoneal cavity using a 23-G needle. A total of 32 rats were subjected to 30 minutes of left coronary artery occlusion, followed by two hours of reperfusion. The rats were allocated into four groups, as follows - (a) sham $+4 \mathrm{~mL}$ of LR group: sham-operated rats in which the left coronary artery was not tightened $(n=8)$; (b) sham + EP50 group: sham-operated rats in which $50 \mathrm{mg} / \mathrm{kg}$ $\mathrm{EP}$ dissolved in $4 \mathrm{~mL}$ of $\mathrm{LR}$ was infused intraperitoneally one hour before the sham operation $(n=8)$; (c) I/R + LR group: rats in which $4 \mathrm{~mL}$ of $L R$ was infused intraperitoneally one hour before the ischaemic offence $(n=8)$; and (d) I/R + EP50 group: rats in which $50 \mathrm{mg} / \mathrm{kg}$ EP dissolved in $4 \mathrm{~mL}$ of $L R$ was infused intraperitoneally one hour before the ischaemic offence $(n=8)$. Vehicle (LR $4 \mathrm{~mL}$ ) or dissolved EP (50 mg/kg) in $4 \mathrm{~mL}$ of LR was infused intraperitoneally one hour before ischaemia. The animals were exposed to 30 minutes of ischaemia followed by two hours of reperfusion, and tissue samples were used for Western blot analysis $(n=4)$ and apoptosis assay $(n=4)$.

Total proteins were taken from the areas at risk (AARs), as described in a previous report. ${ }^{(28)}$ Myocardial tissues were placed in a lysis buffer containing protease inhibitors, homogenised and then centrifuged. After determining the protein concentration in each sample using a modified Bradford assay, 50-80 $\mu \mathrm{g}$ of total protein was heated in a loading buffer. The proteins were separated by $10 \%$ sodium dodecyl sulphate polyacrylamide gel electrophoresis and transferred to nitrocellulose membranes. The membranes were blocked with 5\% skimmed milk and subsequently incubated with primary antibodies against nuclear factor (NF)-кB p65, Bcl-2 (B-cell lymphoma 2 protein), Bax (Bcl-2-associated $X$ protein) and cleaved caspase-3 (Santa Cruz Biotechnology Inc, Santa Cruz, CA, USA) at $4^{\circ} \mathrm{C}$ overnight. The proteins were identified using a horseradish peroxidase-conjugated secondary antibody (Santa Cruz Biotechnology Inc, Santa Cruz, CA, USA) (1:5,000 in Tris-buffered saline and Tween 20 containing 5\% skimmed milk powder for one hour at room temperature) and were visualised by enhanced chemiluminescence. The blots were measured by laser scanning densitometry (GS-670 Imaging Densitometer; Bio-Rad, Manasquan, NJ, USA).

We assessed caspase-3 activity and conducted DNA laddering and terminal deoxynucleotidyl transferase dUTP nick end labelling (TUNEL) staining to explore the anti-apoptotic effect of EP. The activity of caspase-3 was assessed using a colorimetric activity assay kit (Chemicon International Molecular, Temecula, CA, USA), as per the manufacturer's instructions. Briefly, myocardial tissue specimens were taken from the AARs, homogenised in ice-cold buffer (supplied with the kit) and centrifuged. After determining the protein concentration, the supernatant was incubated with caspase-3 substrate (Ac-DEVDpNA) on a 96-well plate. Caspase-3 activity was measured at $405 \mathrm{~nm}$ by spectrophotometry (Infinite ${ }^{\circledR}$ F200; Tecan Group Ltd, Männedorf, Switzerland); the results were seen as fold increases over the mean value of the sham operation.

DNA fragmentation into nucleosomal fragments is the most distinctive biochemical mark of apoptosis. Internucleosomal DNA fragmentation, a recognisable mark of apoptotic cell death, was detected as a DNA laddering pattern using agarose gel electrophoresis. For agarose gel electrophoresis, the frozen tissues (approximately 30-40 mg) were diluted in $500 \mu \mathrm{L}$ of lysis buffer (PUREGENE® DNA Isolation Kit; Gentra Systems Inc, Minneapolis, MN, USA) and quickly homogenised with a microfuge tube pestle. The homogenised tissues were assimilated with $100 \mu \mathrm{g} / \mathrm{mL}$ proteinase $\mathrm{K}$ at $56^{\circ} \mathrm{C}$ for two hours and then incubated with ribonuclease $\mathrm{A}$ at $37^{\circ} \mathrm{C}$ for one hour. After incubation, the tissues were precipitated and centrifuged at $13,000 \times \mathrm{g}$ for five minutes. DNA was precipitated with isopropanol. After centrifugation, the resulting pellets were washed with $75 \%$ ethanol and dissolved in DNA hydration solution (Promega, Madison, WI, USA). After the DNA content was identified at $260 \mathrm{~nm}$ by spectrophotometry (Infinite ${ }^{\circledR}$ F200; Tecan Group Ltd, Männedorf, Switzerland), $10 \mu \mathrm{g}$ of DNA was loaded onto a $1.8 \%$ agarose gel containing $0.5 \mu \mathrm{g} / \mathrm{mL}$ ethidium bromide. Electrophoresis was carried out at $80 \mathrm{~V}$ for one hour. The presence of DNA ladders, which indicate apoptotic nucleosomal DNA fragmentation, were viewed and photographed under ultraviolet transillumination. 
The most common method used to identify apoptosis in tissues is TUNEL staining. For this purpose, tissues were excised from the AARs, fixed in $4 \%$ formaldehyde and coated in paraffin. The tissues were cut into serial sections of $3 \mu \mathrm{m}$ thickness for staining. Immunohistochemical TUNEL staining was used with a commercial kit (Roche Applied Science, Indianapolis, IN, USA). After staining, the sections were tagged with anti-desmin antibodies, counterstained with propidium iodide and evaluated using confocal fluorescence microscopy (FluoView ${ }^{\mathrm{TM}}$ 500; Olympus Optical Co Ltd, Tokyo, Japan). Three sections per sample were randomly adopted and ten microscopic fields per section were assessed. In each field, nuclei were counted and the percentage of TUNEL-positive nuclei was calculated.

All values were shown as mean \pm standard deviation. All statistical analyses were performed using SPSS version 12.0 for Windows (SPSS Inc, Chicago, IL, USA). Differences among groups were analysed using Friedman's one-way analysis of variance and a post-hoc Duncan's multiple comparison test. Statistical significance was defined at $p<0.05$.

\section{RESULTS}

We assessed apoptosis-associated signals using Western blot analysis to explore the effect of EP and its relationship with apoptosis. Our results for $\mathrm{Bcl}-2$, Bax and cleaved caspase- 3 are shown in Fig. 1. Myocardial I/R damage clearly induced Bax and cleaved caspase-3 expressions compared to the sham group. In comparison with the $\mathrm{I} / \mathrm{R}$ control group pretreated with LR alone, $\mathrm{EP}$ significantly increased the expression of $\mathrm{Bcl}-2$ but reduced Bax and cleaved caspase-3 expressions (Fig. 1).

Assessment of caspase-3 activity, and DNA laddering and TUNEL staining to explore the anti-apoptotic effect of EP showed that caspase- 3 activity was decreased in the $\mathrm{I} / \mathrm{R}+\mathrm{EP} 50$ group compared to the I/R + LR group $(146 \% \pm 18 \%$ vs. $185 \% \pm 15 \%$; $\mathrm{p}=0.020$ ) (Fig. 2). Agarose gel electrophoresis to detect DNA laddering patterns found no DNA ladder patterns in the sham + LR or sham + EP50 groups. However, ladders were clearly detected in the $\mathrm{I} / \mathrm{R}+\mathrm{LR}$ group after two hours of reperfusion. The I/R + EP50 group had a significantly reduced display of DNA ladders due to I/R (Fig. 3). TUNEL staining to identify apoptosis in tissues showed that $\mathrm{l} / \mathrm{R}$ damage induced a significant increase in the number of TUNEL-positive cells caused by apoptosis (sham: $5 \% \pm 2 \% ; \mathrm{l} / \mathrm{R}: 41 \% \pm 6 \% ; \mathrm{p}<0.001$ ) (Figs. 4 \& 5). However, in the $\mathrm{I} / \mathrm{R}+\mathrm{EP} 50$ group, the increase in TUNEL-positive cells was significantly less than that in the $\mathrm{I} / \mathrm{R}+\mathrm{LR}$ group $(41 \% \pm 6 \%$ vs. $29 \% \pm 4 \% ; p<0.001)$.

\section{DISCUSSION}

Injection of EP has been shown to improve cardiac function following regional myocardial $\mathrm{I} / \mathrm{R}$ damage in an in vivo rat model. ${ }^{(19)}$ In the present study, we demonstrated that this protective effect may be related, at least in part, to the anti-inflammatory response. ${ }^{(19)}$ We found that the myocardial protective effect of EP may also be related to the prevention of apoptosis. The anti-apoptotic effect of EP was examined with respect to various biochemical variables.

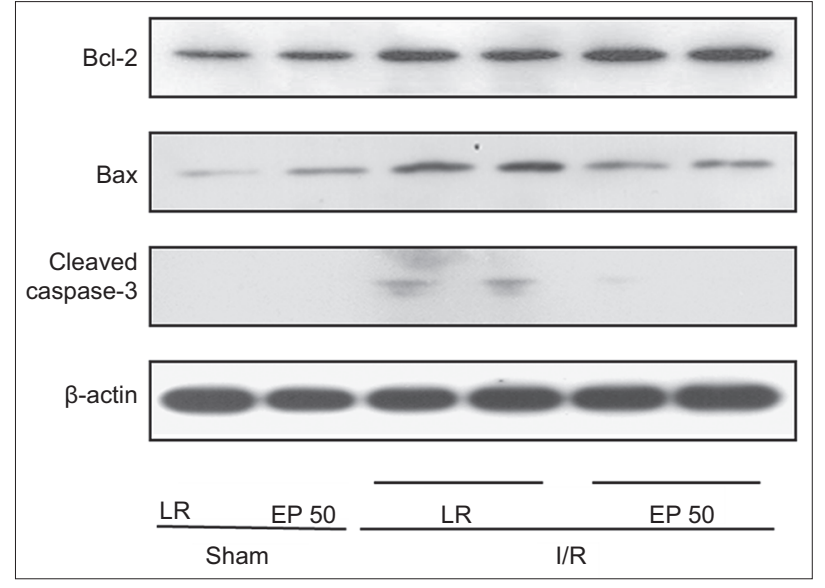

Fig. 1 Western blot analysis of B-cell lymphoma $2(\mathrm{Bcl}-2)$ protein, $\mathrm{Bcl}-2$ associated $X$ (Bax) protein and cleaved caspase- 3 expressions in the areas at risk, in hearts from the sham and ethyl pyruvate (EP)-treated groups. The figure shows four groups with similar results: ischaemia/reperfusion $(\mathrm{I} / \mathrm{R})+$ EP50: rats in which $50 \mathrm{mg} / \mathrm{kg}$ EP dissolved in lactated Ringer's solution (LR) was administered before the ischaemic insult; I/R + LR: rats in which LR was administered before the ischaemic insult; sham + EP50: sham-operated rats in which $50 \mathrm{mg} / \mathrm{kg}$ EP dissolved in LR was administered before the sham operation; sham + LR: sham-operated rats in which LR alone was administered and the left coronary artery was not tightened.

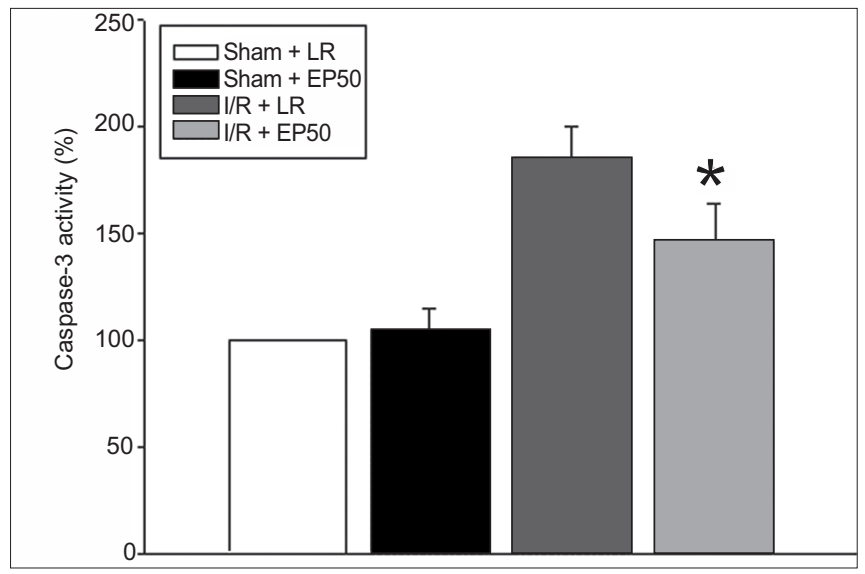

Fig. 2 Graph shows mean caspase-3 activities in the areas at risk in hearts from the sham and ethyl pyruvate (EP)-treated groups ( $n=4$ per group). ${ }^{*} p<0.05$ was statistically significant. I/R: ischaemia/reperfusion; EP50: $50 \mathrm{mg} / \mathrm{kg}$ EP dissolved in $4 \mathrm{~mL}$ of lactated Ringer's solution; LR: lactated Ringer's solution; sham: rats in which no tightening of the left coronary artery was performed

EP has been shown to have a myocardial protective effect in several I/R damage models. ${ }^{(5,14,19)}$ In particular, the myocardial protective effect of EP was proven in regional $I / R$ damage, ${ }^{(19)}$ which is important because regional $I / R$ damage is commonly encountered in the clinical scenario. EP improved cardiac function after regional I/R damage in rats after two hours of reperfusion. It was also reported to be an efficacious anti-apoptotic agent in in vitro and in vivo models. ${ }^{(10,20,24)}$ For instance, a previous study demonstrated that EP has an antiapoptotic effect in an in vitro global myocardial $\mathrm{I} / \mathrm{R}$ damage model. ${ }^{(20)}$ However, it is not clear whether its anti-apoptotic effects are associated with the protective effect in an in vivo regional I/R damage model.

It has been established that cell death following $\mathrm{I} / \mathrm{R}$ damage happens through necrotic and apoptotic pathways. Apoptosis 


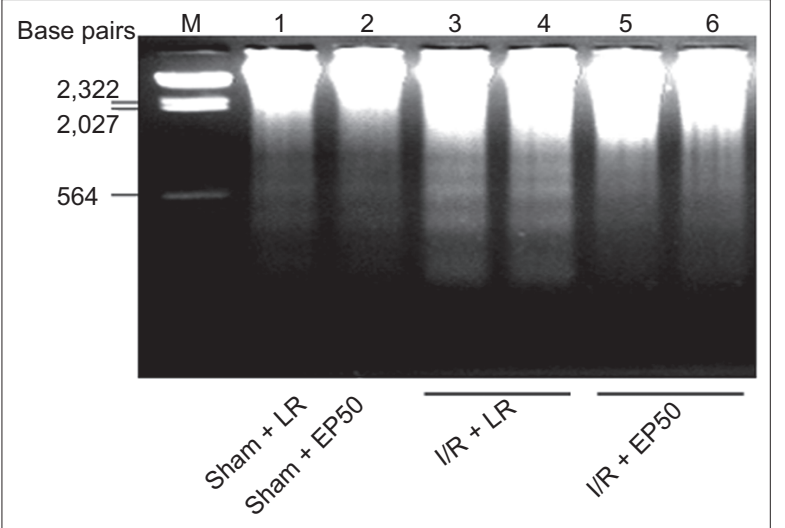

Fig. 3 Detection of internucleosomal DNA fragmentation using agarose gel electrophoresis in the areas at risk in hearts from the sham and ethyl pyruvate (EP)-treated groups. Each lane represents a separate animal. The figure shows four groups with similar results: ischaemia/reperfusion (I/R) + EP50: rats in which 50 mg/kg EP dissolved in lactated Ringer's solution (LR) was administered before the ischaemic insult; I/R + LR: rats in which LR was administered before the ischaemic insult; sham + EP50: sham operated rats in which $50 \mathrm{mg} / \mathrm{kg}$ EP dissolved in LR was administered before the sham operation; sham + LR: sham-operated rats in which LR alone was administered and the left coronary artery was not tightened. Lanes 1 and 2: sham tissue; Lanes 3-6: ischaemic tissue after 30 minutes of ischaemia followed by two hours of reperfusion in the $I / R+L R$ and I/R + EP50 groups; M: marker

has been shown to be involved in the pathogenesis of various cardiovascular diseases and congestive heart failure. ${ }^{(29,30)}$ We validated the finding that $\mathrm{I} / \mathrm{R}$ damage increased the expression levels of the proapoptotic proteins Bax and cleaved caspase-3, but reduced the expression of the anti-apoptotic protein $\mathrm{Bcl}-2$. Overexpression of $\mathrm{BCl}-2$, which is chiefly located on the outer membrane of mitochondria, inhibits the activation of caspase-3 in response to a variety of apoptotic signals. ${ }^{(31)}$ In contrast to I/R damage, EP pretreatment attenuated the increases in Bax and cleaved caspase-3 expression induced by $\mathrm{l} / \mathrm{R}$ damage, while also increasing $\mathrm{Bcl}-2$ expression.

Apoptotic signals bring about the activation of a family of cysteine proteases referred to as caspases. Among them, caspase-3 plays an important role in the mitochondria-dependent apoptosis mechanism. ${ }^{(32)}$ To further investigate the anti-apoptotic effect of EP, we measured caspase-3 activity and confirmed that I/R injury increased caspase-3 activity, whereas EP pretreatment reduced the degree of increase. The protective effect of EP may contribute to repression of the apoptotic reaction through downregulation of caspase- 3 activity.

The present study also demonstrated the anti-apoptotic effect of EP using other biochemical parameters. The reduction of nuclear DNA into nucleosomal segments, which is strong evidence of apoptosis, manifests as DNA laddering caused by the activation of endogenous endonucleases with cleavage of the chromatin DNA into internucleosomal fragments at intervals of approximately 200 base pairs. ${ }^{(33)}$ The fragmentation that is expressed as a laddering pattern is visualised by agarose gel electrophoresis. We found that I/R-induced DNA laddering was reduced by EP pretreatment.

TUNEL staining, the most frequently used technique to observe apoptosis in tissues, labels the ends of DNA strand breaks, which are an indicator of the apoptotic process. ${ }^{(34,35)}$ The TUNEL
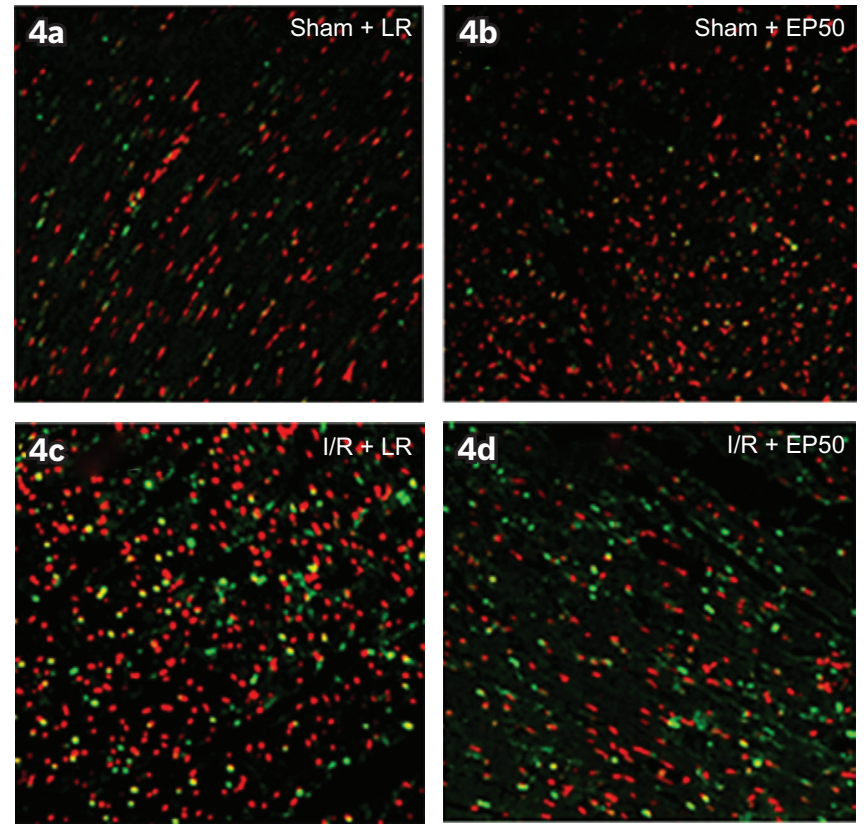

Fig. 4 Double staining images show the detection of apoptotic myocytes using TUNEL and propidium iodide in the areas at risk of hearts from the (a) sham + LR, (b) sham + EP50, (c) I/R + LR, and (d) $1 / R+E P 50$ groups. TUNEL-positive nuclei appear yellow, whereas negative nuclei are stained red by propidium iodide. Original magnifications: $\times 200(a-d)$. I/R: ischaemia/reperfusion; EP50: $50 \mathrm{mg} / \mathrm{kg}$ ethyl pyruvate dissolved in $4 \mathrm{~mL}$ of lactated Ringer's solution; LR: lactated Ringer's solution; sham: rats in which no tightening of the left coronary artery was performed; TUNEL: terminal deoxynucleotidyl transferase dUTP nick end labelling

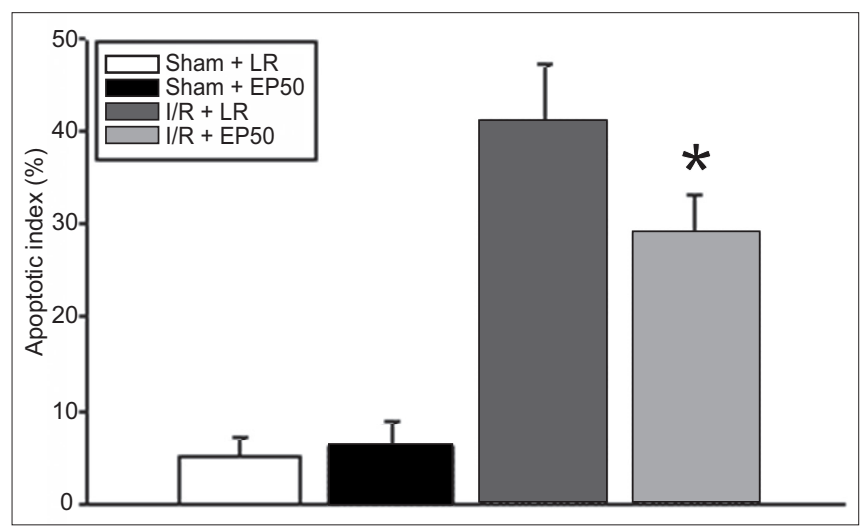

Fig. 5 Graph shows mean percentage of TUNEL-positive nuclei, indicating apoptotic myocytes, detected in the areas at risk in hearts from the sham and ethyl pyruvate (EP)-treated groups ( $n=4$ per group). ${ }^{*} p<0.05$ with respect to the I/R + LR group was statistically significant. I/R: ischaemia/ reperfusion; EP50: $50 \mathrm{mg} / \mathrm{kg}$ EP dissolved in $4 \mathrm{~mL}$ of lactated Ringer's solution; LR: lactated Ringer's solution; sham: rats in which no tightening of the left coronary artery was performed; TUNEL: terminal deoxynucleotidyl transferase dUTP nick end labelling

assay has been used to evaluate the extent of tissue-programmed cell death in both in vitro and ex vivo models. ${ }^{(35)}$ In a previous study, propidium iodide was used as a counterstain to observe TUNEL-negative nuclei together with anti-desmin antibodies in order to quantify apoptosis in myocytes. ${ }^{(34)}$ We found that the increased number of TUNEL-positive cells following myocardial I/R damage was reduced by EP pretreatment in tissue sections.

Our study was not without limitations. First, we used Western blotting to analyse tissues in the AARs from the ischaemic section of the left ventricle. Thus, it is possible that the samples 
collected may have been polluted with endothelial cells, smooth muscle cells and other cell types. Second, we applied EP only at $50 \mathrm{mg} / \mathrm{kg}$. In a future study, a dose-dependent experiment needs to be performed in order to assess the possibility of an additional myocardial protective effect. Third, more experiments need to be conducted to extrapolate our findings to humans.

In conclusion, EP had an anti-apoptotic effect after regional I/R damage in an in vivo rat heart model. The myocardial protective effect of EP may be related to its anti-apoptotic effect.

\section{ACKNOWLEDGEMENTS}

This study was funded by the National Research Foundation of Korea (NRF-2011-0021216) and Biomedical Research Institute fund (GNUHBIF-2016-0004) from Gyeongsang National University Hospital, Jinju-si, Gyeongsangnam-do, South Korea.

\section{REFERENCES}

1. O'Donnell-Tormey J, Nathan CF, Lanks K, DeBoer CJ, de la Harpe J. Secretion of pyruvate. An antioxidant defense of mammalian cells. J Exp Med 1987; 165:500-14.

2. Das UN. Pyruvate is an endogenous anti-inflammatory and anti-oxidant molecule. Med Sci Monit 2006; 12:RA79-84.

3. Das UN. Is pyruvate an endogenous anti-inflammatory molecule? Nutrition 2006; 22:965-72.

4. Raedschelders K, Ansley DM, Chen DD. The cellular and molecular origin of reactive oxygen species generation during myocardial ischemia and reperfusion. Pharmacol Ther 2012; 133:230-55.

5. Dobsak P, Courderot-Masuyer C, Zeller M, et al. Antioxidative properties of pyruvate and protection of the ischemic rat heart during cardioplegia. J Cardiovasc Pharmacol 1999; 34:651-9.

6. Mallet RT, Sun J, Knott EM, Sharma AB, Olivencia-Yurvati AH. Metabolic cardioprotection by pyruvate: recent progress. Exp Biol Med (Maywood) 2005; 230:435-43.

7. Yu YM, Kim JB, Lee KW, et al. Inhibition of the cerebral ischemic injury by ethyl pyruvate with a wide therapeutic window. Stroke 2005; 36:2238-43.

8. Cicalese L, Lee K, Schraut W, et al. Pyruvate prevents ischemia-reperfusion mucosal injury of rat small intestine. Am J Surg 1996; 171:97-100; discussion 100-1.

9. Sileri P, Schena S, Morini S, et al. Pyruvate inhibits hepatic ischemia-reperfusion injury in rats. Transplantation 2001; 72:27-30.

10. Tsung A, Kaizu T, Nakao A, et al. Ethyl pyruvate ameliorates liver ischemiareperfusion injury by decreasing hepatic necrosis and apoptosis. Transplantation 2005; 79:196-204.

11. Lee JY, Kim YH, Koh JY. Protection by pyruvate against transient forebrain ischemia in rats. J Neurosci 2001; 21:RC171.

12. Kung CW, Lee YM, Cheng PY, Peng YJ, Yen MH. Ethyl pyruvate reduces acute lung injury via regulation of iNOS and $\mathrm{HO}-1$ expression in endotoxemic rats. J Surg Res 2011; 167:e323-31.

13. Cruz RJ Jr, Harada T, Sasatomi E, Fink MP. Effects of ethyl pyruvate and other $\alpha$-keto carboxylic acid derivatives in a rat model of multivisceral ischemia and reperfusion. J Surg Res 2011; 165:151-7.

14. Woo YJ, Taylor MD, Cohen JE, et al. Ethyl pyruvate preserves cardiac function and attenuates oxidative injury after prolonged myocardial ischemia. J Thorac
Cardiovasc Surg 2004; 127:1262-9.

15. Taylor MD, Grand TJ, Cohen JE, et al. Ethyl pyruvate enhances ATP levels, reduces oxidative stress and preserves cardiac function in a rat model of offpump coronary bypass. Heart Lung Circ 2005; 14:25-31.

16. Kim JB, Yu YM, Kim SW, Lee JK. Anti-inflammatory mechanism is involved in ethyl pyruvate-mediated efficacious neuroprotection in the postischemic brain. Brain Res 2005; 1060:188-92.

17. Kim HS, Cho IH, Kim JE, et al. Ethyl pyruvate has an anti-inflammatory effect by inhibiting ROS-dependent STAT signaling in activated microglia. Free Radic Biol Med 2008; 45:950-63.

18. Yang R, Han X, Delude RL, Fink MP. Ethyl pyruvate ameliorates acute alcoholinduced liver injury and inflammation in mice. J Lab Clin Med 2003; 142:322-31.

19. Jang IS, Park MY, Shin IW, et al. Ethyl pyruvate has anti-inflammatory and delayed myocardial protective effects after regional ischemia/reperfusion injury. Yonsei Med J 2010; 51:838-44.

20. Guo J, Zhang K, Ji Y, Jiang X, Zuo S. Effects of ethyl pyruvate on myocardial apoptosis and expression of $\mathrm{BCl}-2$ and Bax proteins after ischemia-reperfusion in rats. J Huazhong Univ Sci Technolog Med Sci 2008; 28:281-3.

21. Ulloa L, Ochani M, Yang H, et al. Ethyl pyruvate prevents lethality in mice with established lethal sepsis and systemic inflammation. Proc Natl Acad Sci U S A 2002; 99:12351-6.

22. Fink MP. Ethyl pyruvate. Curr Opin Anaesthesiol 2008; 21:160-7.

23. Zhao ZQ, Nakamura M, Wang NP, et al. Dynamic progression of contractile and endothelial dysfunction and infarct extension in the late phase of reperfusion. J Surg Res 2000; 94:133-44.

24. Mongan PD, Capacchione J, West S, et al. Pyruvate improves redox status and decreases indicators of hepatic apoptosis during hemorrhagic shock in swine. Am J Physiol Heart Circ Physiol 2002; 283:H1634-44.

25. Mongan PD, Fontana JL, Chen R, Bünger R. Intravenous pyruvate prolongs survival during hemorrhagic shock in swine. Am J Physiol 1999; 277(6 Pt 2):H2253-63.

26. National Research Council (US) Institute for Laboratory Animal Research. Guidance for the Description of Animal Research in Scientific Publications. National Academies Press (US): The National Academies Collection, 2011.

27. Shin IW, Jang IS, Lee SH, et al. Propofol has delayed myocardial protective effects after a regional ischemia/reperfusion injury in an in vivo rat heart model. Korean J Anesthesiol 2010; 58:378-82.

28. Jin YC, Kim CW, Kim YM, et al. Cryptotanshinone, a lipophilic compound of Salvia miltiorriza root, inhibits TNF-alpha-induced expression of adhesion molecules in HUVEC and attenuates rat myocardial ischemia/reperfusion injury in vivo. Eur J Pharmacol 2009; 614:91-7.

29. Crow MT, Mani K, Nam YJ, Kitsis RN. The mitochondrial death pathway and cardiac myocyte apoptosis. Circ Res 2004; 95:957-70.

30. Gottlieb RA, Engler RL. Apoptosis in myocardial ischemia-reperfusion. Ann N Y Acad Sci 1999; 874:412-26.

31. Chinnaiyan AM, Orth K, O'Rourke K, et al. Molecular ordering of the cell death pathway. $\mathrm{BCl}-2$ and $\mathrm{BCl}-\mathrm{xL}$ function upstream of the CED-3-like apoptotic proteases. J Biol Chem 1996; 271:4573-6

32. Liu X, Zou H, Slaughter C, Wang X. DFF, a heterodimeric protein that functions downstream of caspase-3 to trigger DNA fragmentation during apoptosis. Cell 1997; 89:175-84.

33. Bortner CD, Oldenburg NB, Cidlowski JA. The role of DNA fragmentation in apoptosis. Trends Cell Biol 1995; 5:21-6.

34. Scarabelli TM, Knight RA, Rayment NB, et al. Quantitative assessment of cardiac myocyte apoptosis in tissue sections using the fluorescence-based tunel technique enhanced with counterstains. J Immunol Methods 1999; 228:23-8.

35. Gavrieli Y, Sherman Y, Ben-Sasson SA. Identification of programmed cell death in situ via specific labeling of nuclear DNA fragmentation. J Cell Biol 1992; 119:493-501. 